GRADIATION\&APPLICATIONS

ISSN 2466-4294 (online) | rad-journal.org

Vol. 3 | Issue 3 | pp. 147-151, 2018

doi: 10.21175/RadJ.2018.03.025

Case study

\title{
STUDY OF THE DISCREPANCY BETWEEN ANALYTICAL CALCULATIONS AND THE OBSERVED BIOLOGICAL EFFECTIVENESS IN PROTON BORON CAPTURE THERAPY (PBCT)
}

\author{
G.A.P. Cirrone ${ }^{1,2}$, G. Petringa ${ }^{1^{*}}$, A. Attili3 ${ }^{3}$, D. Chiappara ${ }^{1,6}$, \\ L. Manti4,5, V. Bravatà7, D. Margarone' ${ }^{2}$, M. Mazzocco6,8, G. Cuttone1 \\ ${ }^{1}$ Laboratori Nazionali del Sud, Istituto Nazionale di Fisica Nucleare, Catania, Italy \\ ${ }^{2}$ Institute of Physics, Czech Academy of Science ELI-Beamlines, Dolní Břežany, Czech Republic \\ 3INFN Section of Roma "Roma Tre", Rome, Italy \\ 4Physics Department, University of Naples Federico II, Naples, Italy \\ 5INFN Naples Section, Complesso Universitario di Monte S. Angelo, Naples, Italy \\ ${ }^{6}$ Dipartimento di Fisica e Astronomia, Università di Padova, Padova, Italy \\ 7Institute of Molecular Bioimaging and Physiology, National Research Council, (IBFM-CNR), Cefalù, Italy \\ 8INFN-Sezione di Padova, Padova, Italy
}

\begin{abstract}
A work recently published experimentally demonstrates an increase in the radiobiological efficacy of clinical proton beams when a tumour is treated in the presence of a concentration of ${ }^{11} B$. For the first time, this paper demonstrates the potential role of the $p{ }^{11} B \rightarrow 3 a($ for brevity, $p-B)$ reaction in the biological enhancement of proton therapy effectiveness. The work reports robust experimental data in terms of clonogenic cell survival and chromosomal aberrations and unambiguously shows the presence of an enhancement when cells were exposed to a clinical proton beam subject to treatment with sodium boroncaptate (BSH). Moreover, the greater occurrence of complex-type chromosomal exchanges points to the effect in terms of radiation of a LET (Linear Energy Transfer) greater than that of protons alone, possibly the alpha particles generated by the reaction. At the same time, we emphasized that analytical calculations, performed on the basis of the well-known total production cross-section data, are not able to explain the effect in a macroscopic way, i.e., solely in terms of a trivial increase in the total dose released in the cells by the alpha-particles. In this paper, thanks to simulations and analytical calculations, we will discuss the theoretically expected alpha-particle yield and the corresponding LET and RBE (Relative Biological Effectiveness) increase related to the ${ }^{11} B$ presence. We conclude that a mere calculation based on the classical concepts of integral dose and average LET and RBE cannot be used to justify the observed radiobiological phenomena. We therefore suggest that micro- and nano-dosimetric aspects must be taken into account.
\end{abstract}

Key words: DMF, proton-Boron enhancement, $P B C T$, proton therapy

\section{INTRODUCTION}

Proton therapy represents one of the fastest growing radiotherapy modalities and one of the most attractive solutions when radiotherapy is needed, even compared to newer and more advanced photon-based techniques such as intensity-modulated radiation therapy (IMRT).

Protons are considered a low-LET (Linear Energy Transfer) radiation, with a clinical Relative Biological Effectiveness (RBE), assumed to be 1.1 (i.e. only ten percent bigger than gamma or $\mathrm{x}$-ray radiation). Such a low biological efficiency is considered a limitation, especially in all those cases where radioresistant tumors must be treated, for which higher $\mathrm{Z}$ ions are preferred (i.e. ${ }^{12} \mathrm{C}$ ions). For these reasons, the proton therapy community is actively investigating the possibility of increasing the biological efficiency of protons by the concomitant use of different drugs and/or other agents to radiosensitize the tumor.
This paper discusses the possibility of taking advantage of the ${ }^{11} \mathrm{~B}$ isotope as an agent able to deliver secondary particles, enhancing the radiobiological effectiveness of protons triggering nuclear reactions.

The clinical proton-boron (p-B) approach was first proposed by Do-Kun et al. [1]. They suggested exploiting the features of the $p\left({ }^{11} \mathrm{~B}, \alpha\right) 2 \alpha$ fusion reaction, where low-energy (about $4 \mathrm{MeV}$ ) alpha particles are generated with a non-negligible production cross-section (around 1.7 barn) for lowenergy (resonance at about $650 \mathrm{keV}$ ) incident protons.

The Korean group also proposed the possibility of using a $718 \mathrm{keV}$ prompt-gamma, emitted in the interaction of protons with ${ }^{11} \mathrm{~B}$ as an imaging source and a potential indicator of the ${ }^{11} \mathrm{~B}$ uptake region. Opposite to this, Giuffrida et al. [2] and Petringa et al. [3] underlined that the gamma emission is not generated from the fusion process but from the elastic $\mathrm{p}\left({ }^{10} \mathrm{~B},{ }^{10} \mathrm{~B}\right.$ ') $\mathrm{p}^{\prime}$ reaction (when using a mixture of ${ }^{10} \mathrm{~B}$ and $\left.{ }^{11} \mathrm{~B}\right)$ and from the $\mathrm{p}\left({ }^{11} \mathrm{~B},{ }^{11} \mathrm{~B}\right) \mathrm{p}$ ' channel and that, in the

* giada.petringa@lns.infn.it 
latter case, at the clinically attainable ${ }^{11} \mathrm{~B}$ concentration in tissues (order of $10^{-5} \mathrm{~g} / \mathrm{cm}^{3}$ ), the peak signal would be completely masked by the non-prompt components generated when protons interact with the natural components of the human body ( ${ }^{16} \mathrm{O}$ mainly).

Experimental results conducted by Petringa et al. [3] have led to finding that in order to maintain the proton-boron therapeutic efficiency and, simultaneously, its imaging capability, the use of boron coupled with natural copper should be adopted [5].

The first experimental proof of radiobiological enhancement obtained by using the $\mathrm{p}-\mathrm{B}$ reaction has been published by Cirrone et al. [4], when they irradiated prostate cancer cells (DU145) and nontumorigenic breast epithelial MCF-10A cell lines along a $62 \mathrm{MeV}$ clinical proton spread out Bragg peak (SOBP) at the Italian ocular proton therapy facility of INFN-LNS, Catania, Italy. Both clonogenic doseresponse curves and complex type chromosomal aberration analyses clearly showed that the presence of Boron nuclei results in an increase of the radiobiological effectiveness of the proton beam. Besides the advantage of using a neutron-free nuclear fusion reaction, the relevance of this method stems from the fact that the reaction cross-section becomes significantly high at relatively low incident proton energy, i.e., around the Bragg peak region. In conventional proton therapy, the beam is typically slowed down inside the tumor (the Bragg peak region). Thus, most of the beam energy (related to the total released dose) is delivered to the tumor cells. Assuming that a given concentration of ${ }^{11} \mathrm{~B}$ nuclei is present preferentially, but not exclusively, in the tumor volume, the incoming slow protons can trigger fusion reaction events and generate highly DNA-damaging alpha particles.

The observed radiobiological enhancement reported in [4], even if confirmed in four experimental sessions, still cannot be explained by making simple use of the knowledge about reaction cross-sections and/or of analytical considerations based on the classical concepts of dose, LET, and RBE.

In this paper, the calculation of alpha particles along the proton SOBP in the irradiation conditions used in [4] will be presented in detail and discussed.

The recent radiobiological experimental results on PBCT are briefly recalled in Section 3 while the estimation of the generated alpha particles along the SOBP and the way these affect the LET distributions are reported in Section 3 and Section 4.

\section{THE RADIOBIOLOGICAL EVIDENCE}

In order to verify the potentiality of the ${ }^{11} \mathrm{~B}(\mathrm{p}, \alpha) 2 \alpha$ reaction for the enhancement of cell-killing in a therapeutic proton therapy scenario, cells from the human prostate cancer DU145 cell line have been irradiated at graded doses $(0.5,1,2,4 \mathrm{~Gy})$ in the middle of a $62-\mathrm{MeV}$ clinical SOBP.

A significant effect due to the boron treatment (dissolved in the medium 6-8 $\mathrm{hr}$ prior to exposure in order to give a concentration of $80 \mathrm{ppm}$ ) was observed throughout four experimental campaigns [4]. Based upon the measured clonogenic survival dose-responses, a Dose Modification Factor (or DMF) of $1.46 \pm 0.12$ was determined at the $10 \%$ survival level. This has suggested that boron atoms could confer a radiobiological advantage in cell-killing

Investigation of structural DNA damages in the form of chromosome aberrations (CAs), a wellacknowledged biomarker of high-LET radiation exposure, subsequently confirmed the presence of high-LET radiation, strongly advocating their contribution to the clonogenic results.

\section{MONTE CARLO SIMULATIONS AND ANALYTICAL EVALUATIONS TO ESTIMATE THE ALPHA YIELD}

Monte Carlo (MC) simulations coupled with analytical calculations were adopted to estimate the total alpha particles produced at the cell site of the experiment reported in Cirrone et al. [4] in the attempt to explain the observed radiobiological enhancement. The proton spectra at various depths in water have been reproduced using $\mathrm{MC}$; these, coupled with the alpha total production cross section, allowed an analytical derivation of the expected alpha particle yield. The total production cross-section was derived from experimental data (using the EXFOR [6] database) and TALYS [7] calculation, when experimental data were not available.

\subsection{Monte Carlo simulations}

Simulations were carried out using the Geant4 (GEometry ANd Tracking) toolkit [8], version 10.04.p02, and the official Geant4 advanced example Hadrontherapy [9]. This example is able to simulate each element of the CATANA (Centro di Adroterapia ed Applicazioni Nucleari Avanzate) passive proton beam line of the INFN-LNS, where the experimental campaigns reported in [4] were performed.

The simulated geometry includes all the transport elements comprising the specific energy modulator for generating the SOBP, the diagnostic elements, and the water T-25 flask where cells were positioned. The latter is simulated through a 40x40x40-mm box, divided into slices of water $100 \mu \mathrm{m}$ in thickness. The slices are perpendicular to the beam axis direction. The number of alpha particles was computed in the slices along the whole SOBP.

All the necessary physics processes were included in the simulations: decay physics, electromagnetic physics, and physics of the hadronic (elastic and nonelastic) interactions. For hadronic processes, the Geant4 QGSP BIC physics list was used [10]. Electromagnetic physics was included in the activation of the G4EmStandardPhysics_opt4 physics list, enabling high accuracy in tracking the electrons, hadrons and ions [11].

A total of $1 \mathrm{M}$ proton histories have been tracked in each simulation run.

The simulated energy spectra of the primary proton beam are reported in Figure 1 at four different depths along the SOBP (0.0 mm, 19.0 mm, 25.0 mm, $29.4 \mathrm{~mm}$ in water). 
G.A.P. Cirrone et al., Proton boron capture therapy (PBCT), Rad. Applic., 2018, 3, 3, 147-151

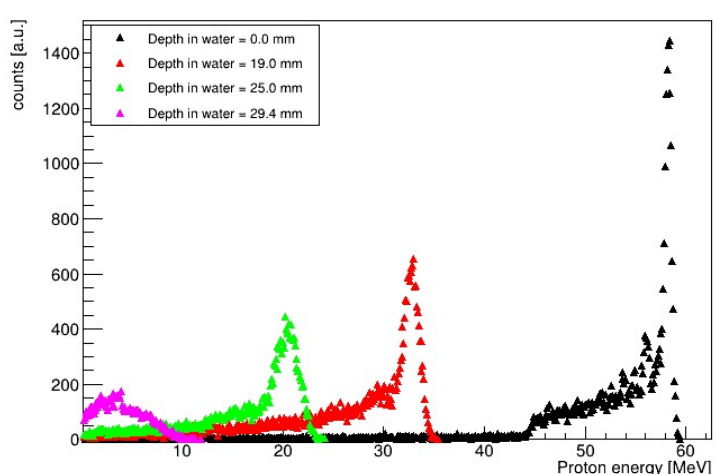

Figure 1. Proton energy spectra at various depths inside the water tank

\subsection{Alpha production cross-sections}

The cross sections of the proton-boron total production are needed for the computation of the expected number of produced alphas. The TALYS-1.9 analytical code [7] was used to retrieve them due to its ability to account for all different production channels induced by protons.

At low energies (below $10 \mathrm{MeV}$ ), the ${ }^{11} \mathrm{~B}(\mathrm{p}, \alpha) 2 \alpha$ channel is the only one producing alpha particles; experimental cross-sections for this channel are also available and data can be found in the EXFOR database [6]. Figure 2 shows the ${ }^{11} \mathrm{~B}(\mathrm{p}, \quad \alpha) 2 \alpha$ experimental cross section and the total TALYS production cross section results, summing all reactions producing alpha particles. A factor to account for alpha multiplicity corresponding to every given reaction is taken into account.

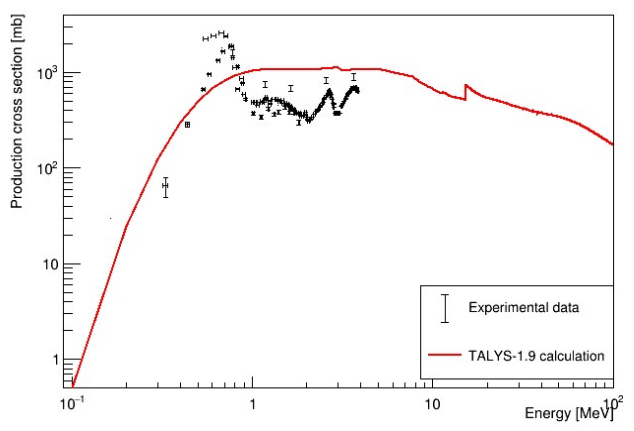

Figure 2. Experimental ${ }^{11} \mathrm{~B}(\mathrm{p}, \mathrm{a}) 2 \alpha$ and TALYS total production cross-section vs. energy of the incident proton

Considering that TALYS poorly describes the data below $1 \mathrm{MeV}$, we decided to adopt a combination of the two distributions as a total cross-section: the experimental data below $4 \mathrm{MeV}$ and the TALYS calculations above that energy.

\subsection{Alpha estimation}

A quantification of alpha particles generated at any depth $\left(\mathrm{N}_{\alpha}\right)$ along the SOBP due to the ${ }^{11} \mathrm{~B}$ presence was performed by embedding the simulated proton spectra of Figure 1 in the equation:

$$
\frac{d N_{\alpha}}{d N_{p}}=\sigma_{\alpha} n_{x} d
$$

where $\sigma_{\alpha}$ is the total alpha production cross-section derived from the union of the TALYS and experimental data, $\mathrm{n}_{\mathrm{x}}$ is expressed in atoms $/ \mathrm{m}^{3}\left(\mathrm{n}_{\mathrm{x}}=3.56 \cdot 10^{24}\right.$ atoms $/ \mathrm{m}^{3}$ corresponding to the ${ }^{11} \mathrm{~B}$ concentration used), and $d$ is the voxel size (100 $\mu \mathrm{m})$.

The ${ }^{11} \mathrm{~B}$ numerical density, $\mathrm{n}_{\mathrm{x}}$, was estimated using the following equation:

$$
n_{x}=f \frac{C_{B} \rho_{t o t} N_{a}}{M_{B}}
$$

where $C_{B}$ is the mass concentration of boron (8o ppm in our case), $\rho$ tot is the total density of the target (in this case it has been taken as equal to that of water) $\mathrm{N}_{\mathrm{A}}$ is the Avogadro constant, $M_{B}$ is the atomic mass of boron and $\mathrm{f}$ is the fraction of ${ }^{11} \mathrm{~B}$ with respect to the total natural boron amount introduced into the cell culture through the BSH agent (here a fraction of 0.8 has been considered).

The final number of alpha particles is calculated by scaling the total number of protons, $\mathrm{N}_{\mathrm{x}}$, in each slide in order to obtain a dose of $2 \mathrm{~Gy}$ in the given position.

\section{DISCUSSION}

The average Linear Energy Transfer (or LET) can be retrieved by considering only the primary proton spectra along the SOBP (Figure 1) and using the formula:

$$
L_{D}=\frac{\sum L_{i} \varepsilon_{i}}{\sum \varepsilon_{i}}
$$

Here, $\mathrm{L}_{\mathrm{i}}$ is the ratio between the energy deposited by the incident proton with an energy of $\varepsilon_{\mathrm{i}}$ and a step length of $\mathrm{L}_{\mathrm{i}}$ [12].

The same definition of Equation 3 can be adopted to estimate the LET along the SOBP, also including the contribution of the generated alphas' $\mathrm{n}_{\mathrm{x}}$.

For this purpose, only the alpha coming from ${ }^{11} \mathrm{~B}(\mathrm{p}$, a) $2 \alpha$ channel as been used, as their spectrum is well known, and that channel is the one the authors of [4] use to justify the biological effect.

After the inclusion of the generated alphas, the calculated LET variation appears, on the one hand, to be negligible and it can be shown that, in order to obtain a DMF as the one experimentally observed, a LET of the order of 4-30 keV/ $\mu \mathrm{m}$ should be observed.

On the other hand, these LET values can be obtained only with a number of alpha particles larger by a factor of $10^{5}$ with respect to those here calculated. In Table 1, the proton LET, the total LET (including the generated alpha particles), and the corresponding DMF are reported for three different positions along the SOBP, also considering an additional alpha particles contribution which is larger by a factor of $10^{5}$ with respect to the values here calculated. The calculated 
G.A.P. Cirrone et al., Proton boron capture therapy (PBCT), Rad. Applic., 2018, 3, 3, 147-151

DMF values are, in this case, able to explain experimental data.

Table 1. DMF estimation (last column) at three different depths along the SOBP; proton dose-averaged LET, LET considering the yield of alpha particles as calculated in this paper and LET with the yield necessary to explain experimental data.

\begin{tabular}{|l|c|c|c|c|}
\hline $\begin{array}{c}\text { Position } \\
{[\mathrm{mm}]}\end{array}$ & $\begin{array}{c}\text { LET of } \\
\text { primary } \\
\text { protons } \\
{[\mathrm{keV} / \mu \mathrm{m}]}\end{array}$ & $\begin{array}{c}\text { LET } \\
\text { considering } \\
\text { calculated } \\
\text { alpha yield } \\
{[\mathrm{keV} / \mu \mathrm{m}]}\end{array}$ & $\begin{array}{c}\text { LET to } \\
\text { explain } \\
\text { experimental } \\
\text { DMF } \\
{[\mathrm{keV} / \mu \mathrm{m}]}\end{array}$ & $\begin{array}{c}\text { Experimental } \\
\text { Dose } \\
\text { Modifying } \\
\text { Factor (DMF) }\end{array}$ \\
\hline $\begin{array}{l}\text { Entrance } \\
(5.0)\end{array}$ & 1.30 & 1.30 & 3.66 & $1.17 \pm 0.37$ \\
\hline $\begin{array}{l}\text { Mid- } \\
\text { SOBP } \\
(21.0)\end{array}$ & 2.29 & 2.29 & 9.53 & $1.36 \pm 0.44$ \\
\hline $\begin{array}{l}\text { Distal } \\
(29.8)\end{array}$ & 11.70 & 11.70 & 33.92 & $2.04 \pm 0.66$ \\
\hline
\end{tabular}

LET values are also reported in Figure 1, where the gap between calculated LET and necessary one is shown.

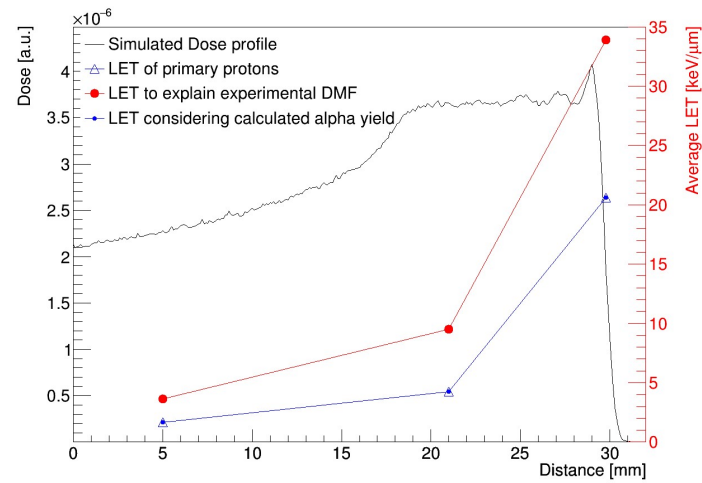

Figure 3. LET due to protons, considering calculated alpha yield and able to describe experimental DMF

\section{CONCLUSION}

Inelastic reactions triggered by energetic protons as they slow down across a clinical SOBP interacting with boron nuclei seem to produce an enhancement of proton radiobiological effectiveness. These results, even if experimentally well-corroborated by repeated experimental campaigns [4], seem to fall short of an immediate justification by estimations merely based on analytical and Monte Carlo evaluations of classical dosimetric quantities such as integral dose, LET, and RBE.

The total number of alpha particles generated and estimated on the basis of the well-known total production cross-section of the $\mathrm{p}-\mathrm{B}$ reaction, does not explain the experimental results in terms of an average LET increase and, hence, with a corresponding RBE enhancement. On the other hand, if classical radiobiological models are applied, in order to reach the observed DMF values, one would require a number of alpha particles larger by a factor of $10^{5}$ with respect to the calculated yield.
In the future, a micro and nano dosimetric approach should be taken into account as it may give experimental information about the number and quality of high-LET particles produced, and may also take into account the biological effects of a local huge dose deposit able to explain part of the discrepancy.

Different biological effects, like the creation of free radicals and bystander effect, could also contribute to the explanation of the effect experimentally observed.

Acknowledgements: This paper has been written in the framework of the Move-it, MC-INFN, the NEPTUNE projects funded by the Committee $V$ of Italian Institute for Nuclear Physics (INFN) and of the GAMA project (TGo2010056) of ELI-Beamlines facility (Dolnì Brezany, CZ).

\section{REFERENCES}

1. D-K. Yoon, J-Y. Jung, T. S. Suh, "Application of proton boron fusion reaction to radiation therapy: A Monte Carlo simulation study," Appl. Phys. Lett., vol. 105, no. 22, 223507, 2014, Dec. 2014.

DOI: $10.1063 / 1.4903345$

2. L. Giuffrida et al., "Prompt gamma ray diagnostics and enhanced hadron-therapy using neutron-free nuclear reactions," AIP Advances, vol. 6, no. 10, pp. 105 - 204, Oct. 2016.

DOI: 10.1063/1.4965254

3. G. Petringa et al., "Study of gamma-ray emission by proton beam interaction with injected boron atoms for future medical imaging applications," J. Instrumentation, vol. 12, no. 3, Co3049, Mar. 2017. DOI: $10.1088 / 1748-0221 / 12 / 03 / \mathrm{Co} 3049$

4. G. A. P. Cirrone et al., "First experimental proof of Proton Boron Capture Therapy (PBCT) to enhance protontherapy effectiveness," Sci. Rep., vol. 8, 1141, Jan. 2018.

DOI: $10.1038 / \mathrm{s} 41598-018-19258-5$

PMid: 29348437

PMCid: PMC5773549

5. S. Xuan et al., "Synthesis and in vitro studies of a series of carborane-containing boron dipyrromethenes (bodipys)," J. Med. Chem. vol. 59, no. 5, pp. 2109 2117, Feb. 2016

DOI: 10.1021/acs.jmedchem.5bo1783

PMid: 26849474 PMCid: PMC4893941

6. N. Otuka et al., "Towards a More Complete and Accurate Experimental Nuclear Reaction Data Library (EXFOR): International Collaboration Between Nuclear Reaction Data Centres (NRDC)," Nucl. Data Sheets, vol. 120, pp. $272-276$, Jun. 2014. DOI: $10.1016 /$ j.nds.2014.07.065

7. A. Koning et al., "Modern Nuclear Data Evaluation with the TALYS Code System," Nucl. Data Sheets, vol. 113, no. 12, pp. 2841 - 2934, Dec. 2012. DOI: 10.1016/j.nds.2012.11.002

8. S. Agostinelli et al., "Geant4-a simulation toolkit," Nucl. Instrum. Methods A, vol. 506 pp. 250 - 303, 2003. DOI: 10.1016/So168-9002(03)01368-8

9. G. A. P. Cirrone et al., "Hadrontherapy: a Geant4-Based Tool for Proton/Ion-Therapy-studies," Prog. Nucl. Sci. Technol., vol. 2, pp. 207 - 212, 2011.

DOI: 10.15669/pnst.2.207

10. Geant4, A Simulation Toolkit: Physics Reference Manual Release 10.4, CERN, Geneva, Switzerland, 2017.

Retrieved from: http://geant4userdoc.web.cern.ch/geant4- 
G.A.P. Cirrone et al., Proton boron capture therapy (PBCT), Rad. Applic., 2018, 3, 3, 147-151

userdoc/UsersGuides/PhysicsReferenceManual/fo/Phy sicsReferenceManual.pdf;

Retrieved on: Aug. 10, 2018

11. Physics List EM constructors in Geant4 10.4, CERN, Geneva, Switzerland, 2018.

Retrieved from: https://geant4.web.cern.ch/node/1731 \#opt4;

Retrieved on: Aug. 10, 2018
12. D. Sanchez-Parcherisa et al., "Analytical calculation of proton linear energy transfer in voxelized geometries including secondary protons," Phys. Med. Biol., vol. 61, no. 4, pp. 1705 - 1721, Feb. 2016.

DOI: $10.1088 / 0031-9155 / 61 / 4 / 1705$

PMid: 26840945 